The prevalence of autism spectrum traits and autism spectrum disorders in children and adolescents with obsessive compulsive disorder: a systematic review

Claire Tiley ${ }^{1 \star}$ and Marinos Kyriakopoulos ${ }^{2}$

${ }^{1}$ South London and Maudsley NHS Foundation Trust and ${ }^{2}$ South London and Maudsley NHS Foundation Trust, Institute of Psychiatry, Psychology and Neuroscience, King's College London

${ }^{*}$ Corresponding author.

doi: 10.1192/bjo.2021.194

Aims. Autism Spectrum Disorder (ASD) and Obsessive Compulsive Disorder (OCD) commonly co-occur in children and adolescents (C\&A); evidence suggests functional impairment is increased in those diagnosed with both disorders. The aims of this systematic review were: 1) To review studies that report on the prevalence of ASD traits and/or diagnosis in C\&A with OCD. 2) To review whether the severity of OCD symptoms is related to the severity of ASD traits in C\&A with OCD. 3)To review whether the severity of comorbid ASD traits or diagnosis in C\&A with OCD impact on their global functioning.

Method. This systematic review was registered in PROSPERO. Prisma guidelines were followed . Electronic searches were carried out on Pubmed, EMBASE and Psychinfo with the use of selected keywords. Inclusion criteria : 1) Participants up to the age of 18 who had an ICD or DSM diagnosis of OCD. 2) Journal articles published in the English, with no date specifications. 3) Papers evaluating ASD diagnosis or traits, or where data on this could be extracted. Exclusion criteria: 1) Papers looking at OCD related disorders such as body dysmorphic disorder, compulsive skin picking, trichotillomania and hoarding disorder. 2) Samples including adults where C\&A data could not be extracted. 3) Posters, abstracts and dissertations.

Result. A total of 15 studies were included in the systematic review. Seven of these studies directly compared the prevalence of ASD traits (measured by questionnaires) or diagnosis in OCD to a control group or normative data, with all studies reporting a significant elevation in ASD trait scores and diagnosis in OCD. Ten of the studies reported on the correlation between ASD trait severity and OCD severity. Four studies identified a significant correlation between ASD and OCD total scores or specified subscales. In contrast, one study found significantly elevated OCD scores in an OCD only group when compared to a comorbid OCD and ASD group. Three studies reported on the correlation between ASD scores and functional impairment or compared an OCD only group to a comorbid group. All three studies demonstrated that the presence ASD or ASD traits are associated with elevated scores in global functional impairment. Conclusion. In conclusion, this review suggests that there is an increased prevalence of ASD traits and diagnosis amongst C\&A with OCD. Elevated ASD traits within this population are associated with a greater impact on global functioning.

Four-six year clinical follow-up of deep brain stimulation for obsessive compulsive disorder

Himanshu Tyagi

UCL Institute of Neurology, Queen Square, The National Hospital for Neurology and Neurosurgery

doi: 10.1192/bjo.2021.195

Background. Over the past 20 years a number of robust studies have established the clinical effectiveness and safety of Deep brain stimulation (DBS) in adults with profound multi-treatmentrefractory obsessive-compulsive disorder (OCD). However long term (>12 months) outcomes with this novel neurosurgical intervention are still inadequately reported. Our group conducted the first UK study of DBS in OCD between 2013-2017. All participants in our trial achieved a responder status at 15 month endpoint and the main results were reported in 2019. A specialist multidisciplinary clinic was established after the trial to provide life-long aftercare in the form of scheduled clinical and hardware reviews. Here we are reporting a preliminary analysis of the long-term clinical, functional and social outcomes from this cohort.

Method. Long term follow-up clinical data (15-75 months, 2015 onwards) were prospectively collected from the participants who were enrolled in the original MRC-UCL pilot study of DBS for OCD. DBS parameters, battery health and status, social circumstances, mental state and medication adjustments were noted alongside the outcome measures of YBOCS at clinical follow-up encounters. Additional ratings of GAF, SDS and certain qualitative measures were recorded at least once every year since initial study completion. Result. Five out of six participants continued with DBS treatment and kept responder status. One participant had his DBS switched off and hardware removed. One participant had multiple hospital admissions to manage comorbidity progression to primary condition. One participant had OCD severity scores revised upwards despite continuing gains in QoL. Secondary outcomes generally matched the 15 month end point of initial trial. All participants experienced minor to major changes in their relationships with partners or family. Qualitative feedback indicated that DBS was well tolerated by $5 / 6$ subjects but the burden of specialist follow-up remained significant.

Conclusion. Our long term follow-up data indicate that DBS is safe and conferred a sustained long-term benefit in reduction of obsessive-compulsive symptoms. A non-trivial burden of checking and maintenance of implanted hardware, comorbidity-unmasking following successful OCD treatment, perceived 'burden of normality' by the participant, need for life-long follow-ups with specialist multidisciplinary team including DBS nurses, highly specialist psychiatrists from National OCD service, neuropsychiatrists, neurologists and neurosurgeons partially counterbalances the gains offered by this treatment. Overall DBS offers a safe, effective and enduring alternative to participants who do not respond to any other form of OCD treatment and do not wish to undergo ablation surgery.

\section{Prevalence of psychosocial distress in school going adolescents in rural Pakistan: findings from a cross-sectional epidemiological survey}

Syed Usman Hamdani ${ }^{1 \star}$, Zill-e- Huma ${ }^{2}$, Hashim Javed ${ }^{3}$, Azza Warraitch ${ }^{4}$, Atif Rahman ${ }^{5}$, Asad Tameezuddin Nizami ${ }^{6}$ and Fareed Aslam Minhas ${ }^{7}$

${ }^{1}$ Institute of Psychiatry, Benazir Bhutto Hospital, Department of Primary Care and Mental Health, University of Liverpool, Global Institute of Human Development, Shifa Tameer-e-Millat University, Human Development Research Foundation; ${ }^{2}$ Department of Primary Care, University of Liverpool, Global Institute of Human Development, Shifa Tameer-e-Millat University, Human Development Research Foundation; ${ }^{3}$ Global Institute of Human Development, Shifa Tameer-e-Millat, University, Human Development Research Foundation; ${ }^{4}$ Human Development Research Foundation; ${ }^{5}$ Department of Primary Care and Mental Health, University of Liverpool; ${ }^{6}$ Institute of Psychiatry, Benazir Bhutto Hospital and ${ }^{7}$ Global Institute of Human Development, Shifa Tameer-e-Millat University

${ }^{\star}$ Corresponding author.

doi: 10.1192/bjo.2021.196 